\title{
Efficient algorithms for the regularization of dynamic inverse problems: II. Applications
}

\author{
U Schmitt $^{1}$, A K Louis ${ }^{1}$, C Wolters ${ }^{2,3}$ and M Vauhkonen ${ }^{4}$ \\ ${ }^{1}$ Institute for Applied Mathematics, PO Box 151150, Saarland University, 66041 Saarbrücken, \\ Germany \\ 2 Max-Planck Institute for Mathematics in the Sciences, Inselstr. 22, 04103 Leipzig, Germany \\ ${ }^{3}$ Max-Planck Institute of Cognitive Neuroscience, PO Box 500355, 04303 Leipzig, Germany \\ ${ }^{4}$ Department of Applied Physics, University of Kuopio, PO Box 1627, 70211, Kuopio, Finland \\ E-mail: schmitt@num.uni-sb.de
}

Received 11 September 2001, in final form 14 February 2002

Published 24 April 2002

Online at stacks.iop.org/IP/18/659

\begin{abstract}
In the first part of this paper the notion of dynamic inverse problems was introduced and two procedures, namely STR and STR-C, for the efficient spatiotemporal regularization of such problems were developed.

In this part the application of the new methods to three practically important problems, namely dynamic computerized tomography, dynamic electrical impedance tomography and spatio-temporal current density reconstructions will be presented. Dynamic reconstructions will be carried out in simulated objects which show the quality of the methods and the efficiency of the solution process. A comparison to a Kalman smoother approach will be given for dynEIT.
\end{abstract}

\section{Introduction}

In the first part [1] of this paper we introduced the notion of dynamic inverse problems and developed two efficient procedures for spatio-temporal regularization, namely STR and STR-C. We will now give a brief repetition of the key facts appearing in the first part.

The starting point is a measuring procedure which needs a certain amount of time. During this time span, measurements are taken at timesteps $t_{i}$. A dynamic problem is then described by equations $A_{i} x_{i}=y_{i}$, where $i$ is a temporal index, i.e. the linear operator $A_{i}$ maps the properties $x_{i}$ of an investigated object to the measurements $y_{i}$ at timestep $t_{i}$.

Since the operators $A_{i}$ are under-determined in most cases, the degree of freedom in $A_{i} x_{i}=y_{i}$ is very high. Due to this fact, and due to the ill-posedness of these equations, we consider the a priori information 'temporal smoothness' which is introduced as follows: 
in order to achieve a solution with the desired properties we expand the well known TikhonovPhillips minimization task with an additional term which measures the total variance of $x_{i}$. Thus, we solve

$$
\Phi(x)=\sum_{i=1}^{T}\left\|A_{i} x_{i}-y_{i}\right\|^{2}+\lambda^{2} \sum_{i=1}^{T}\left\|x_{i}\right\|^{2}+\mu^{2} \sum_{i} \frac{\left\|x_{i+1}-x_{i}\right\|^{2}}{\left(t_{i+1}-t_{i}\right)^{2}} \rightarrow \min .
$$

If we introduce

$$
D=\left(\begin{array}{ccccc}
\frac{1}{t_{2}-t_{1}} & -\frac{1}{t_{2}-t_{1}} & & & \\
& \frac{1}{t_{3}-t_{2}} & -\frac{1}{t_{3}-t_{2}} & & \\
& & \ddots & \ddots & \\
& & & \frac{1}{t_{T}-t_{T-1}} & -\frac{1}{t_{T}-t_{T-1}}
\end{array}\right) \in \mathbb{R}^{T \times(T-1)}
$$

the efficient solution of this minimization task is given by the following procedure named STR [1]:

(i) Input: data $y$, spatial regularization parameter $\lambda$, temporal regularization parameter $\mu$.

(ii) Calculate $Q$ and $R$

$$
\begin{aligned}
& Q=\left(D D^{\top}+\frac{\lambda^{2}}{\mu^{2}} I_{T-1}\right)^{-1} \\
& R=I_{T}-D^{\top} Q D=\left(r_{i, j}\right)_{i, j} .
\end{aligned}
$$

(iii) $C$ is defined by

$$
C=\left[r_{i, j} A_{i} A_{j}^{*}\right]_{i, j} .
$$

(iv) Solve

$$
\left(C+\lambda^{2} I_{G_{1} \oplus \cdots \oplus G_{T}}\right) u=y .
$$

(v) Finally the $x_{i}$ are calculated by

$$
x_{i}=\sum_{j} r_{i, j} A_{j}^{*} u_{j}
$$

In order to achieve efficiency, in a first step one calculates $A_{j}^{*} u_{j}$ for each $j$ and afterwards $x_{i}$.

As this procedure is formulated in terms of operator equations, which may map between infinite-dimensional spaces, one needs a discretization scheme for their numerical solution, see [1] for further information.

If the linear operators $A_{i}$ are identical for all timesteps and if these operators are matrices, one can enhance the efficiency by using the following procedure STR-C: 
(i) Input: data $y$, spatial regularization parameter $\lambda$, temporal regularization parameter $\mu$.

(ii) Calculate $Q$ and $R$ according to

$$
\begin{aligned}
& Q=\left(D D^{\top}+\frac{\lambda^{2}}{\mu^{2}} I_{T-1}\right)^{-1} \\
& R=I_{T}-D^{\top} Q D .
\end{aligned}
$$

(iii) Solve the generalized Sylvester equation

$$
\left(A_{0} A_{0}^{*}\right) U R+\lambda^{2} U=\operatorname{Mat}_{n}(y)=: Y .
$$

(iv) Calculate

$$
X=A_{0}^{*} U R,
$$

and get $x_{i}$ as the $i$ th column of $X$.

The equation in step (iii) is an equation of Sylvester type, an efficient solution algorithm can be found in [2].

\section{Dynamic computerized tomography}

In the following the problem of dynamic computerized tomography (dynCT) will be studied. We will describe how procedure STR can be applied and some numerical tests will be carried out. An overview of the mathematics of computerized tomography can be found in [3-5].

\subsection{Application of procedure STR}

dynCT is a linear problem which will be formulated as operator equations between infinitedimensional Hilbert spaces.

We consider parallel geometry, i.e. the forward problem is described as follows: first

$$
L(s, \omega)=\left\{x \in \mathbb{R}^{2} \mid s-x \cdot \omega=0\right\} \quad \omega \in S^{1}, s \geqslant 0
$$

is defined. Thus, $L$ is a line orthogonal to $\omega$ with distance $s$ to the origin. The Radon transform $\mathcal{R}$ is then defined by

$$
\begin{aligned}
& \mathcal{R}: L^{2}(\Omega) \rightarrow L^{2}\left([-1,1], S^{1}\right) \\
& \mathcal{R} f(s, \omega)=\int_{L(s, \omega)} f(x) \mathrm{d} x=\int_{\Omega} \delta(s-x \cdot \omega) f(x) \mathrm{d} x
\end{aligned}
$$

and

$$
\mathcal{R}_{i} f(s)=\mathcal{R}_{\theta_{i}} f(s)=\mathcal{R} f\left(s, \omega_{i}\right)
$$

according to $\omega_{i}=\left(-\sin \theta_{i}, \cos \theta_{i}\right)^{\top}, 1 \leqslant i \leqslant T$, and $\Omega=\{x \mid\|x\| \leqslant 1\}$. We assume that $\theta_{i}$ are equidistant, $0 \leqslant \theta_{i}<2 \pi$. So $\mathcal{R}_{i}$ maps the density $f_{i}$ of the scanned object at a time $t_{i}$ to the measurements at this point of time. Due to the variation of $\theta$ during a fixed period of time, we get a dynamic problem. 
In order to apply the procedure STR to this problem, we have to determine the weights $r_{i, j}$ as described in step (ii) of STR. As the angles are equispaced, we assume

$$
D=\left(\begin{array}{ccccc}
1 & -1 & & & \\
& 1 & -1 & & \\
& & \ddots & \ddots & \\
& & & 1 & -1
\end{array}\right)
$$

Now, $R=\left(r_{i, j}\right)$ can be computed as described in step (ii) of STR.

Then, we have to consider the operator

$$
C=\left[r_{i, j} A_{i} A_{j}^{*}\right]
$$

with $A_{i}=\mathcal{R}_{\theta_{i}}=\mathcal{R}_{i}$. So $C$ maps from $\left(L^{2}([-1,1])\right)^{T}$ to itself. It is easy to show that

$$
\mathcal{R}_{i}^{*} g(x)=g\left(x \cdot \omega_{i}\right) .
$$

That is, $\mathcal{R}_{i}^{*}$ extends the function $g$ on the detector to a function on the object $\Omega$, such that $\mathcal{R}_{i}^{*} g$ is constant along lines $L\left(s, \omega_{i}\right)$. Due to geometric invariances of the Radon transform [4] we have

$$
\mathcal{R}_{i} \mathcal{R}_{j}^{*}=\mathcal{R}_{(i-j) \bmod T} \mathcal{R}_{0}^{*}
$$

and if we define

$$
C_{i}=\mathcal{R}_{i} \mathcal{R}_{0}^{*}
$$

$[C]$ can be written as

$$
[C]=\left[r_{i, j} C_{(i-j) \bmod T}\right] .
$$

If we want to solve step (iv) in STR numerically by a projection scheme, we discretize the $C_{i}$ and weight and compound the emerging matrices according to lemma 4.1 and (2) to a discretization of $[C]$.

For the discretization of $C_{i}$ we start from angles

$$
\theta_{i}=2 \pi \frac{i-1}{T}, \quad 1 \leqslant i \leqslant T
$$

and $N$ detector elements at

$$
s_{j}=\frac{2 j-1-N}{N-1}
$$

such that $s_{1}=-1$ and $s_{N}=1$. If we now use pointwise linear basic functions $\phi_{k}$ on the detector, defined by $\phi_{k}\left(s_{j}\right)=\delta_{j, k}$ and point collocations $\psi_{k}=\delta_{s_{k}}$, we get a discrete version $\tilde{C}_{i} \in \mathbb{R}^{N \times N}$ of $C_{i}$ by

$$
\left(\tilde{C}_{i}\right)_{j, l}=\psi_{j} C_{i} \phi_{l}=\left(\mathcal{R}_{i} \mathcal{R}_{0}^{*} \phi_{l}\right)\left(s_{j}\right) .
$$

That is, one has to extend the 'hat'-function $\phi_{i}$ to the object $\Omega$, such that the extension is constant along lines parallel to the $x$-axis. Next, one has to calculate the line integral of this function along the line $L\left(s_{j}, \omega_{i}\right)$ (see figure 1). In detail: first, we have to calculate the points

$$
\begin{aligned}
& p_{1}=L\left(s_{l-1}, \omega_{0}\right) \cap L\left(s_{k}, \omega_{i}\right) \quad p_{2}=L\left(s_{l}, \omega_{0}\right) \cap L\left(s_{k}, \omega_{i}\right) \\
& p_{3}=L\left(s_{l+1}, \omega_{0}\right) \cap L\left(s_{k}, \omega_{i}\right) .
\end{aligned}
$$

Then, we determine linear functions $\alpha_{i}$ such that

$$
\alpha_{1}\left(p_{1}\right)=0 \quad \alpha_{1}\left(p_{2}\right)=1 \quad \alpha_{2}\left(p_{2}\right)=1 \quad \alpha_{2}\left(p_{3}\right)=0 .
$$




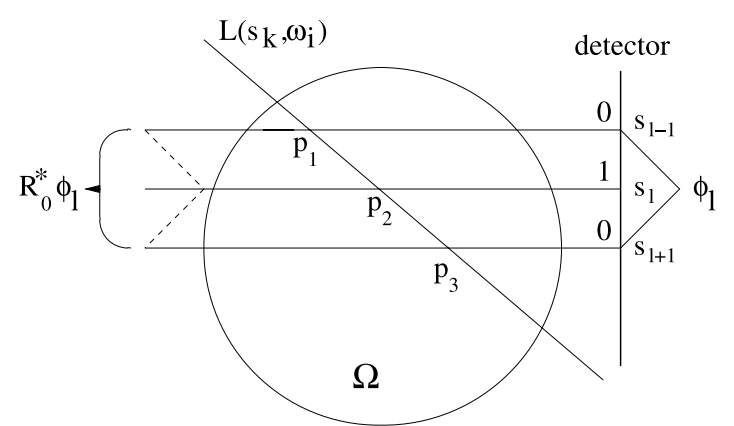

Figure 1. Calculation of the entries of $\tilde{C}_{i}$.

Now

$$
\left(\tilde{C}_{i}\right)_{k, l}=\int_{p_{1}}^{p_{2}} \alpha_{1}(x) \mathrm{d} x+\int_{p_{2}}^{p_{3}} \alpha_{2}(x) \mathrm{d} x .
$$

Furthermore, we have to consider several subcases: the points $p_{i}$ may be outside the object, the points $p_{i}$ may not exist, or the linecuts in (3) may be whole lines, not just points. These are technical details which are handled accordingly.

Step (iv) of STR now delivers vectors $u_{i}$ and corresponding piecewise linear functions $\tilde{u}_{i}=\sum_{j}\left(u_{i}\right)_{j} \phi_{j}$.

In order to complete the implementation of STR we have to compute step (v) as follows. First we divide $[-1,1]$ into $n$ points $z_{i}=\frac{2 i-n-1}{n-1}$, such that $z_{1}=-1$ and $z_{n}=1$. Then we construct a grid $G_{n}$ by

$$
G_{n}=\left\{\left(z_{i}, z_{j}\right) \mid 1 \leqslant i, j \leqslant n\right\}
$$

The resulting dynamical solutions $f_{i}$ of $\mathcal{R}_{i} f_{i}=g_{i}$ will be computed according to step (v) of STR in points $p \in G_{n} \cap \Omega$ by

$$
f_{i}(p)=\sum_{j} r_{i, j}\left(R_{j}^{*} \tilde{u}_{j}\right)(p)=\sum_{j} r_{i, j} \tilde{u}_{j}\left(p \cdot \omega_{j}\right)
$$

In order to evaluate this formula in an efficient way, we first compute functions $U_{j}$ on $G_{n}$ using

$$
U_{j}(p)=\tilde{u}_{j}\left(p \cdot \omega_{j}\right)
$$

and then compute the sum

$$
f_{i}(p)=\sum_{j} r_{i, j} U_{j}(p)
$$


To summarize, we get the following algorithm:

(i) Input: data $g$, spatial regularization parameter $\lambda$, temporal regularization parameter $\mu$.

(ii) Starting with $D, \lambda, \mu$ compute $Q, R$ according to

$$
\begin{aligned}
& Q=\left(D D^{\top}+\frac{\lambda^{2}}{\mu^{2}} I_{T-1}\right)^{-1} \\
& R=I_{T}-D^{\top} Q D=\left(r_{i, j}\right)_{i, j} .
\end{aligned}
$$

(iii) Compute matrices $\left(\tilde{C}_{i}\right)_{j, l}=\psi_{j} C_{i} \phi_{l}=\left(\mathcal{R}_{i} \mathcal{R}_{0}^{*} \phi_{l}\right)\left(s_{j}\right)$. Then, $\tilde{C}$ is compounded according to

$$
\tilde{C}=\left(r_{i, j} \tilde{C}_{(i-j) \bmod T}\right) \in \mathbb{R}^{N T \times N T} .
$$

(iv) Solve

$$
\left(\tilde{C}+\lambda^{2} I_{N T}\right) u=g .
$$

with $u=\left(u_{1}^{\top}, \ldots, u_{T}^{\top}\right)^{\top}$ and $g=\left(g_{1}^{\top}, \ldots, g_{T}^{\top}\right)^{\top}$

(v) Discretize $[-1,1]^{2}$ using a grid $G_{n}$ and compute

$$
U_{j}(p)= \begin{cases}\tilde{u}_{j}\left(p \cdot \omega_{j}\right) & \text { if } p \in \Omega \cap G_{n}, \\ 0 & \text { else. }\end{cases}
$$

Compute $\tilde{u}_{j}(s)$ by linear interpolation of $u_{j}$.

Finally, the solution of dynCT is achieved by using

$$
f_{i}(p)=\sum_{j} r_{i, j} U_{j}(p)
$$

with $p \in G_{n}$.

Step (iv) in this procedure corresponds to a linear system on the detector and step (v) is a weighted and discrete version of the backprojection $\mathcal{R}^{\sharp} \tilde{u}_{j}$, see [4,5].

If we had used stepwise constant functions on the detector and functionals

$$
\psi_{l}(f)=\int_{s_{l-1 / 2}}^{s_{l+1 / 2}} f(x) \mathrm{d} x
$$

we would have obtained a dynamic version of the direct algebraic method as proposed in [4].

\subsection{Numerical tests}

We will see two numerical tests based on the procedure STR applied to dynCT.

The design parameters are as follows: we used a $300 \times 300$ grid for reconstruction, that is $n=300$. Furthermore, we had 87 angular positions, which means we considered $T=87$ timesteps. The detector is divided into 81 points, which means $N=81$. We determined $\lambda=0.01$ and $\mu=1.0$ by experimentation. The data were generated analytically, no noise was added.

The two dynamic objects, that will be studied, have the same structure, see figure 2 . The circular objects are static and the emphasized ellipse is dynamic. 


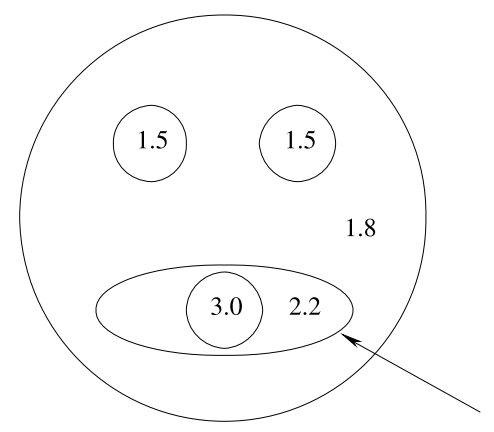

Figure 2. The structure of the considered dynamic objects. The numbers describe the density of the objects, the highlighted ellipse is the dynamic part of the object.
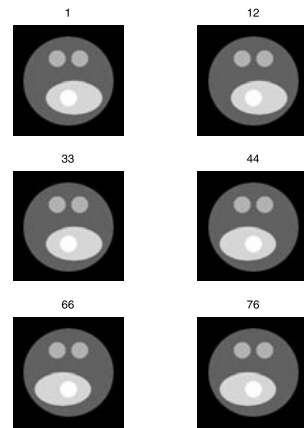
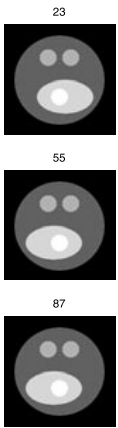
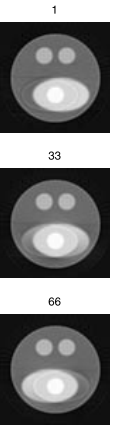
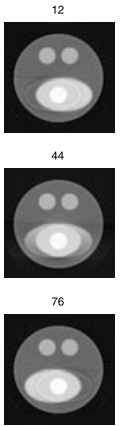
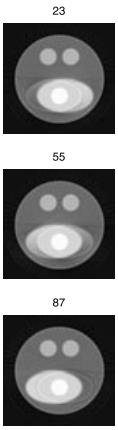

Figure 3. Dynamic object number one and reconstructions. In the left part one can see the original dynamic object, in the right part the reconstructions are depicted. The numbers above the single pictures are the corresponding timesteps.

2.2.1. First example. The first example considers the case of slight patient motion: the examined organs behave statically up to timestep 44 and the ellipse lies on the right-hand side. Then, from timestep 45 on, the ellipse lies on the left-hand side, the other organs do not move. The object and the according reconstructions are shown in figure 3. At the beginning and at the end of the scanning process the quality of the reconstruction is quite good, in the neighbourhood of timestep 43 the reconstructions are blurred and show slight artefacts. Nevertheless one can see the underlying dynamics.

2.2.2. Second example. In this case we examine a dynamic object where the size of the ellipse increases from timestep one to timestep 87. This corresponds to a CT at the human heart or to a CT of the lung during inhalation. The object and the according reconstructions can be seen in figure 4.

2.2.3. Discussion. As we have seen the described method is able to reveal dynamics in a scanned object. The reconstructions are afflicted with artefacts which correspond to the weak a priori information we considered. Nevertheless, the method is able to distinguish between pathological findings (which should appear in reconstructions belonging to all timesteps) and motion artefacts (which are supposed to appear in reconstructions according to few timesteps only). Reconstruction procedures which deliver only one picture do not have this property. Thus, a practical application of our method is imaginable. 

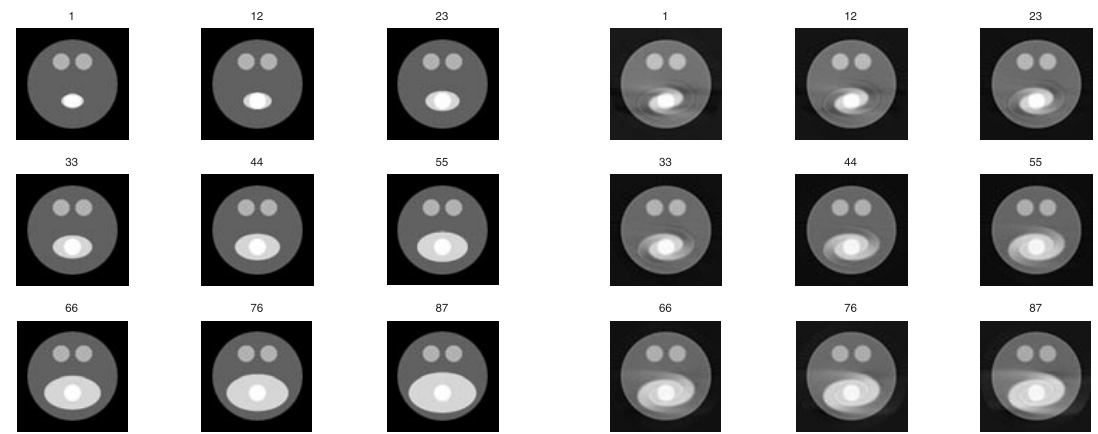

Figure 4. Dynamic object number two and reconstructions. In the left part one can see the original dynamic object, in the right part the reconstructions are depicted. The numbers above the single pictures are the corresponding timesteps.

\section{Dynamic electrical impedance tomography}

Electrical impedance tomography (EIT) tries to determine the conductivity inside a given object based on electrical measurements on the objects surface. For applications see [6-10]. Article [11] gives an extensive survey of EIT.

EIT is based on a nonlinear ill-posed problem that can be interpreted as a dynamic problem, which we will name as dynEIT in the following. In the first section we will give some preliminaries and we will apply the procedure STR to the problem of dynEIT by linearization of the underlying problem. In a second section we will give some numerical tests which we will compare to a known procedure based on so-called (fixed interval) Kalman smoothers.

\subsection{Application of the procedure STR}

The electrical measurements addressed above consist of measured voltages based on injected current patterns. Due to the variation of the currents from timestep to timestep, one can interpret EIT as a dynamic problem dynEIT. We assume that the timesteps are equispaced.

Our calculations are based on a so-called complete electrode model which is proposed in [12]. The outstanding quality of this model is studied in [13]. It is formulated by using the following equations:

$$
\begin{aligned}
& \operatorname{div}(\sigma \nabla u)=0 \\
& \int_{e_{l}} \sigma \frac{\partial u}{\partial n} \mathrm{~d} S=I_{l} \quad 1 \leqslant l \leqslant L \\
& \left.\sigma \frac{\partial u}{\partial n}\right|_{\partial \Omega \backslash \cup e_{l}}=0 \\
& \left.\left(u+z_{l} \sigma \frac{\partial u}{\partial n}\right)\right|_{e_{l}}=U_{l} \quad 1 \leqslant l \leqslant L .
\end{aligned}
$$

Here, $L$ is the number of electrodes, $I_{l}$ is the injected current at electrode $e_{l}, U_{l}$ is the measured voltage at this electrode, $\sigma$ is the conductivity distribution, $u$ is the electrical potential inside the examined object $\Omega, z_{l}$ is a so-called contact impedance at electrode $e_{l}$ and $n$ is the outward normal at $\partial \Omega$.

This equation can be solved by converting it to a variational equation which can be solved by a finite element method (FEM), see [13-16]. The uniqueness of the solution induces a 
nonlinear operator in form of a real valued matrix

$$
T:\left(\rho, I_{l}\right) \mapsto U
$$

Here $\rho=\left(\rho_{i}\right)_{i}$ and $\sum_{i} \rho_{i} \chi_{i}$ is a discretized version of $\rho=1 / \sigma . U$ is a vector of the measured voltages. $\chi_{i}$ are characteristic functions of elements $\Delta_{i}$.

In order to consider EIT as a dynamic problem, operators

$$
A_{t}(\rho)=T\left(\rho,\left(I_{l}\right)_{t}\right), 1 \leqslant t \leqslant T
$$

are used which are linearized in $\rho^{0}$ as follows:

$$
A_{t}(\rho)=A_{t}\left(\rho^{0}\right)+J_{t}\left(\rho^{0}\right)\left(\rho-\rho^{0}\right)+\mathrm{o}\left(\left\|\rho-\rho^{0}\right\|\right) .
$$

The matrix $J_{t}\left(\rho^{0}\right)$ can be computed using the stiffness matrix of the underlying FEM, see [14-16]. $\rho^{0} \in \mathbb{R}$ can be estimated from the data according to [14-16]. In the following, $\rho^{0}$ is either a real number, or we identify $\rho^{0}$ with the vector $\rho^{0}(1, \ldots, 1)$. The length of this vector is arbitrary.

The starting point for the solution of dynEIT are $T$ measurement vectors $U_{i}=\left(U_{k}\right)_{i}, 1 \leqslant$ $k \leqslant L, 1 \leqslant i \leqslant T$ together with the minimization task

$$
\Phi(\rho)=\|A(\rho)-U\|^{2}+\lambda^{2} \sum_{t}\left\|\rho-\rho^{0}\right\|^{2}+\mu^{2}\|B \rho\|^{2} \rightarrow \min
$$

with the notions

$$
\begin{aligned}
& \rho=\left(\rho_{1}^{\top}, \ldots, \rho_{T}^{\top}\right)^{\top} \\
& U=\left(U_{1}^{\top}, \ldots, U_{T}^{\top}\right)^{\top} \\
& A=\operatorname{diag}\left(A_{t}\right) \\
& B=\left[\begin{array}{ccccc}
I & -I & & & \\
& I & -I & & \\
& & \ddots & \ddots & \\
& & & I & -I
\end{array}\right] \text {. }
\end{aligned}
$$

If we now use

$$
J(\rho)=\operatorname{diag}\left(J_{t}\left(\rho_{t}\right)\right)
$$

and approximate $A$ iteratively by linearization we get the following Gauß-Newton type iteration:

$$
\rho^{i+1}=\operatorname{minarg}_{\rho}\left\{\left\|A\left(\rho^{i}\right)+J\left(\rho^{i}\right)\left(\rho-\rho^{i}\right)-U\right\|^{2}+\lambda^{2}\left\|\rho-\rho^{0}\right\|^{2}+\mu\|B \rho\|^{2}\right\} .
$$

The upper indices are iteration indices. It has to be pointed out, that this minimization problem cannot be tackled by the procedure STR due to the spatial regularization term $\left\|\rho-\rho^{0}\right\|$. If we want to use procedure STR, we have to introduce $u=\rho-\rho^{0}$ and $u^{i}=\rho^{i}-\rho^{0}$. If we consider $B u=B \rho$ we get the iteration

$$
u^{i+1}=\operatorname{minarg}_{u}\left\{\left\|J\left(\rho^{i}\right) u-\left(U+J\left(\rho^{i}\right) u^{i}-A\left(\rho^{i}\right)\right)\right\|^{2}+\lambda^{2}\|u\|^{2}+\mu^{2}\|B u\|^{2}\right\}
$$

respectively

$\rho^{i+1}=\rho^{0}+\operatorname{minarg}_{u}\left\{\left\|J\left(\rho^{i}\right) u-\left(U+J\left(\rho^{i}\right)\left(\rho^{i}-\rho^{0}\right)-A\left(\rho^{i}\right)\right)\right\|^{2}+\lambda^{2}\|u\|^{2}+\mu^{2}\|B u\|^{2}\right\}$.

This iteration now has the right mode so that STR can be applied. 

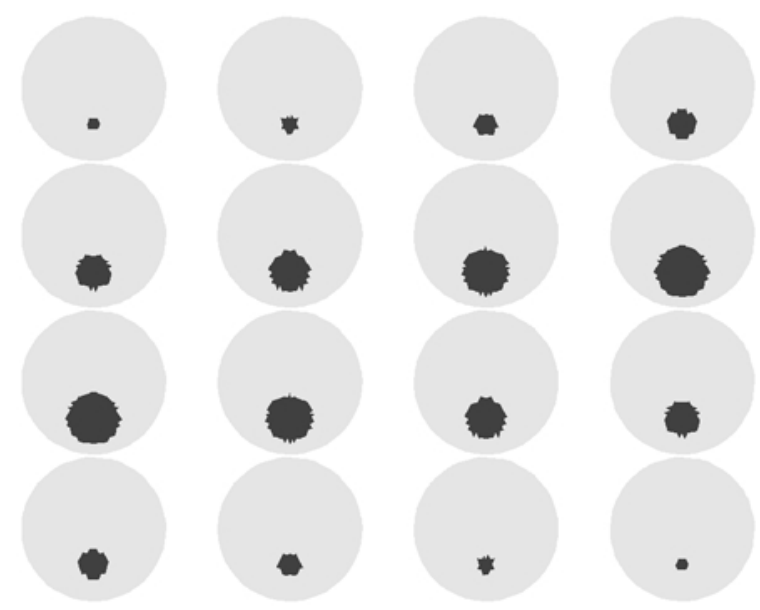

Figure 5. The examined dynamic object.

\subsection{Numerical tests}

For the testing of our proposed method we used synthetic data calculated by a FEM using 1968 elements for the discretization of the unit disc. Furthermore, we used $L=16$ electrodes and $T=16$ current patterns. For the discretization of the resistivities $\rho^{i}$ we used a coarser mesh with 492 elements. The examined object is drawn in figure 5 . The background has the resistivity $400 \Omega$, the inclusions $200 \Omega$. $\rho^{0}$ was estimated [14-16] as $\rho^{0}=396 \Omega$. This object reflects the rise of a bubble in a three-dimensional tube through a two-dimensional plane. In the following we will compare our method with a so-called (fixed interval) Kalman smoother approach. According to $[15,17]$, we choose the identity matrix as the regularization operator. Further details can be found in these two references. For the theory of Kalman smoothers see [18].

It should be pointed out that in the reconstruction results the contrast was modified in order to achieve meaningful images.

3.2.1. Noiseless data. We will now present three different reconstructions: our proposed method with one iteration, see figure 6, our proposed method with two iterations, see figure 7 and the Kalman smoother method, see figure 8. All regularization parameters where chosen by experimentation. The reconstructions of the Kalman smoother have a slightly better quality than the reconstructions achieved by one iteration of our procedure. The quality after two iterations is again enhanced. More iterations do not result in improved reconstructions. After the precalculation of $J\left(\rho^{0}\right)$, the Kalman smoother needs about $80 \mathrm{~s}$ on a Pentium-II-CPU, one iteration of our method needs about $1 \mathrm{~s}$ and two iterations need $60 \mathrm{~s}$. The leap in the running time between one and two iterations is caused by the calculation of the linearization $J\left(\rho^{1}\right)$ in the second iteration of our procedure which depends on the result of the first iteration and thus cannot be precalculated. The memory usage of the Kalman smoother is about 80 times higher than the usage of our iterative method.

3.2.2. Noisy data. Now we superpose our synthetic data with uniform noise in the range $[-0.025 \cdot \max |U|,+0.025 \cdot \max |U|]$. The reconstructions can be seen in figures 9 and 10 . Again, the Kalman smoother reconstructions seem to have a comparable quality to the results 


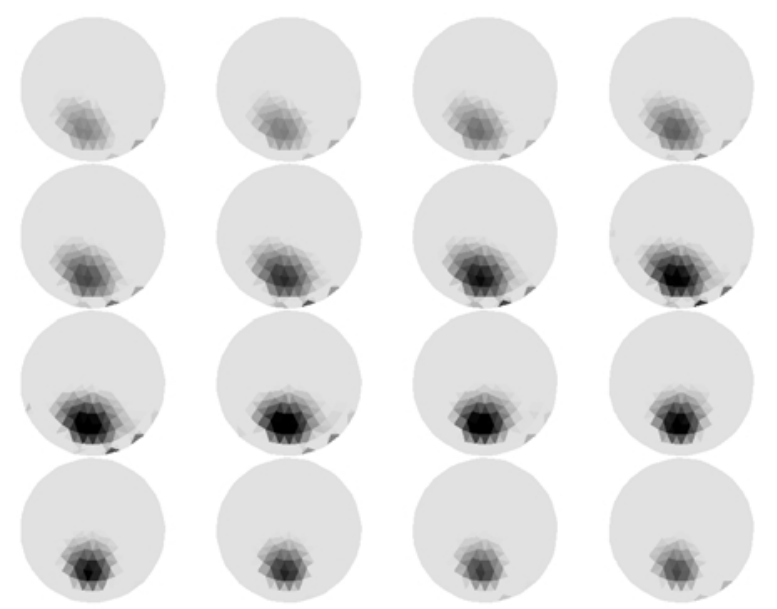

Figure 6. Reconstruction after one iteration of our procedure based on noise-free synthetic data. $\lambda=0.001, \mu=0.0055$.

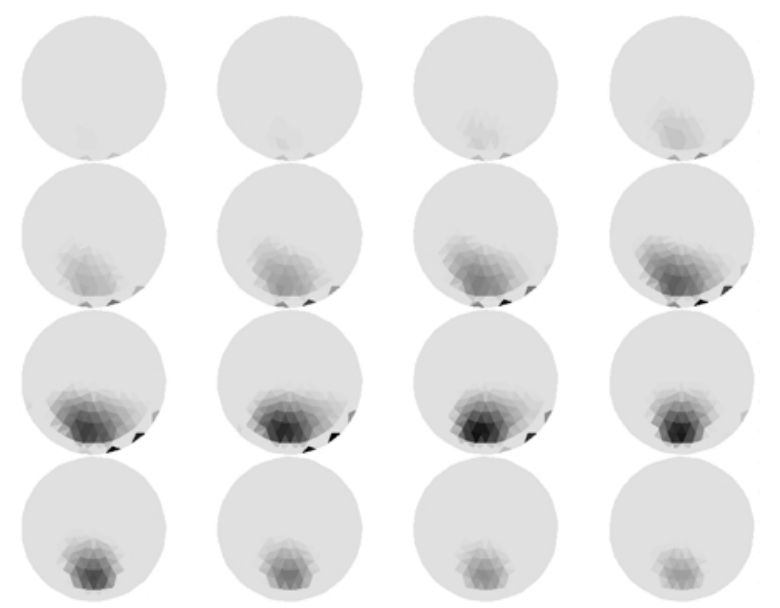

Figure 7. Reconstruction after two iterations of our procedure based on noise-free synthetic data. $\lambda=0.014, \mu=0.005$.

of our approach. According to the results in 3.2.1 one further iteration of our procedure leads to slightly better reconstructions.

\subsection{Discussion}

We have seen that our iterative approach based on STR leads to reconstructions with a satisfying quality. The quality seams to be comparable to the quality of the reconstructions gained by the Kalman smoother.

There is one important difference between the two approaches which has an impact on the practical use: the Kalman smoother is controlled by three parameters, the STR approach only needs two of them. As we have already mentioned, the Kalman approach is significantly slower and has a much higher memory usage.

It should be annotated, that there is another approach called fixed-lag smoothing [19] which has less memory consumption than the (fixed-interval) Kalman smoother used here. 


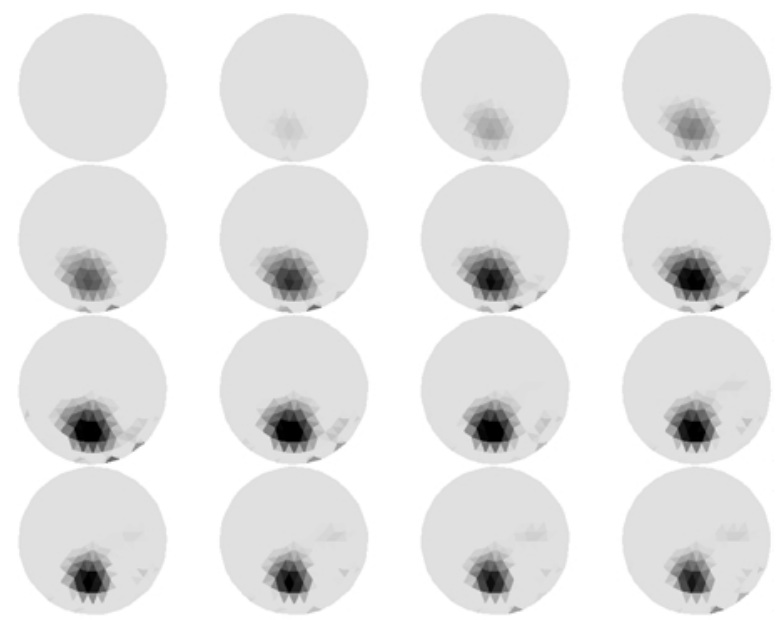

Figure 8. A Kalman smoother reconstruction based on noise-free synthetic data. According to $[15,17]$ we used $\alpha=0, a_{1}=30, a_{2}=0.00001$.
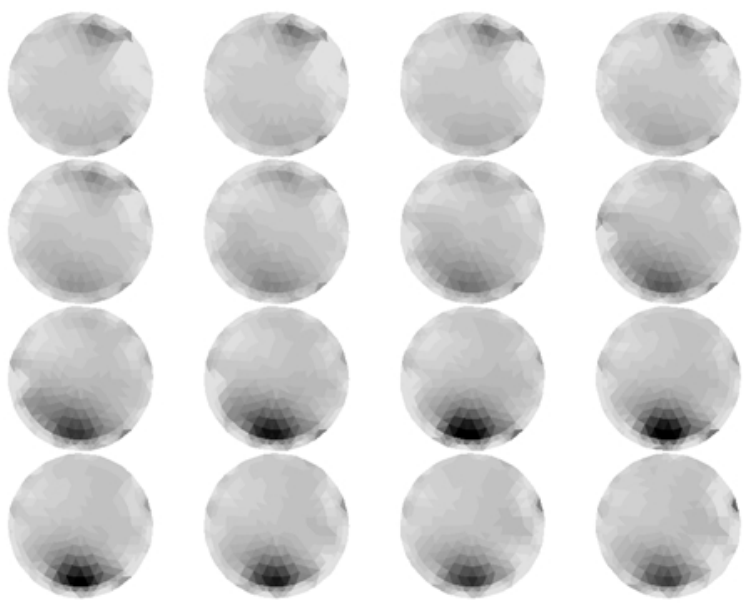

Figure 9. Reconstruction after one iteration based on noisy data. $\lambda=0.02, \mu=0.07$.

The extended Kalman filter studied in [20], is an analogue to our Gauß-Newton approach. This method is supposed to work better than the Kalman smoother in certain cases.

\section{Spatio-temporal current density reconstructions}

Current density reconstructions (CDR) appear in the field of inverse source localizations. Here, one tries to determine electrical activity in an object by electrical measurements on the surface of the object.

One field of application is the study of neurological activity in the human brain, by means of electroencephalography (EEG) measurements on the surface of the head. Focal epileptogenic discharges [21] or sources, underlying somato sensoric evoked potentials (SEPs) can be localized [22].

An overview of the broad field applications is given in [23] and [24]. Comparable techniques can be applied to the human heart, see [25]. 

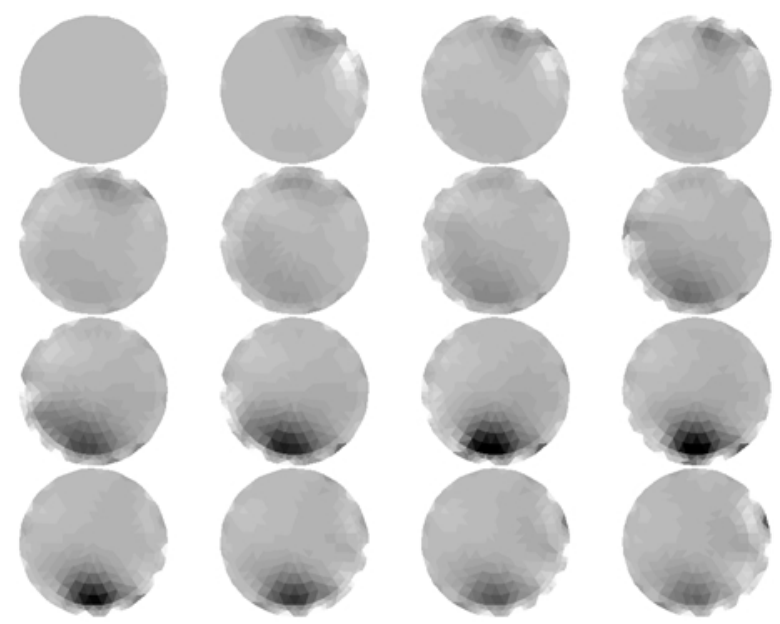

Figure 10. Kalman smoother reconstruction based on noisy data. $\alpha=0.08, a_{1}=30, a_{2}=0.08$.

\subsection{The forward model}

The fundamental equation governing the interaction of electrical sources $j$ and the electrical potential $\Phi$ is the Poisson equation in connection with a Neumann boundary condition

$$
\begin{array}{ll}
\operatorname{div}(\sigma \nabla \Phi)=\operatorname{div} j & \text { in } \Omega \\
\langle\sigma \nabla \Phi, n\rangle=0 & \text { at } \Gamma=\partial \Omega .
\end{array}
$$

Here, $\sigma$ is the conductivity tensor and the open and bounded set $\Omega$ describes the geometry of the head. $n$ is the outward normal at $\partial \Omega$. We define $\Gamma_{0} \subset \partial \Omega$ as the measurement surface. Then, the electrical measurements are obtained as $\Phi \mid \Gamma_{0}$.

In order to achieve a discrete forward model, the current in the object $\Omega$ is discretized as a fixed number of dipoles, located at points $p_{i} \in \Omega, 1 \leqslant i \leqslant N$, and point collocation at the measurement points are used. It is assumed that the measurements are taken at points $\xi_{i} \in \Gamma_{0}$. The set $\left\{p_{i} \mid 1 \leqslant i \leqslant N\right\}$ is called influence space.

If we name the $k$ th unit vector in $\mathbb{R}^{3}$ as $\bar{e}_{k}$, and if we set $e_{i, k}$ as a dipole in $p_{i}$ with moment $\bar{e}_{k}$, that is $e_{i, k}=\delta_{p_{i}} \bar{e}_{k}, j$ can be discretized by

$$
j=\sum_{i=1}^{N} \sum_{k=0}^{2} \alpha_{i+k N} e_{i, k} .
$$

The forward model of CDR is the so-called leadfield matrix. Depending on the geometrical model of the head, this matrix can be computed by analytical formulae [26,27], boundary element methods [28] or FEMs [29]. A fast-forward solution in realistically shaped anisotropic FE head models is described in [30]. In [31] methods were described how $\Omega$ and especially tensor valued $\sigma$ can be determined non-invasively and individually through multi-modal magnetic resonance imaging.

The leadfield matrix maps a current distribution $j$ described by a vector $\alpha$ to the electrical measurements by $m=L \alpha$. For details see [23]. The determination of $\alpha$ is typical for the CDR methods in contrast to dipole fit methods, where only some few dipoles explaining the measured data are determined through an optimization procedure, see [32].

The data are given as functions of time, as provided by an EEG. Most existing CDR methods use separate time slices of voltage measurements without temporal coupling of neighbouring 


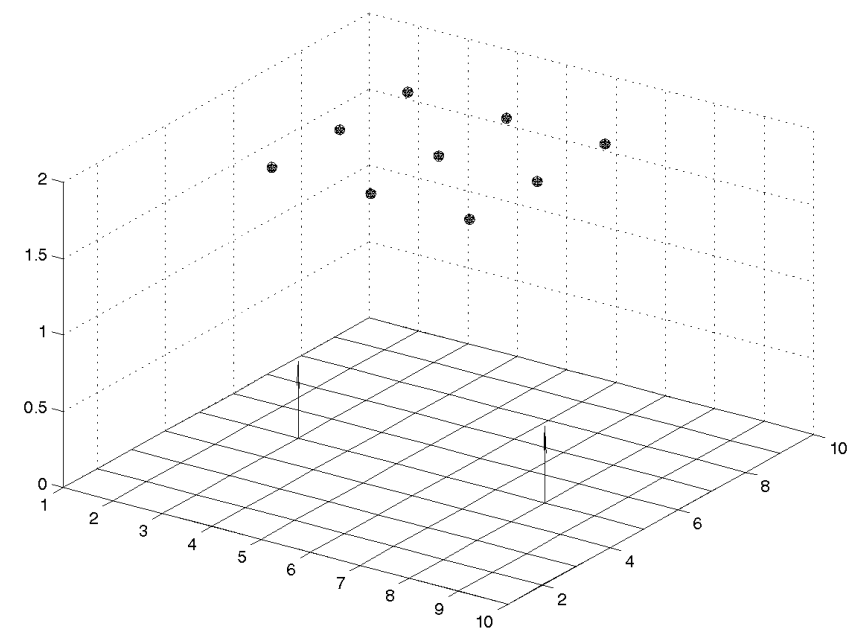

Figure 11. Sketch of the model.

timesteps. Due to the physiologically motivated a priori information of temporal smoothness, it makes sense to use our procedures in order to achieve stability in the presence of noise. As the leadfield matrix is independent of time, we use procedure STR-C.

\subsection{A simple volume conductor model}

The set-up of the model is as follows: a two-dimensional influence space consisting of a $10 \times 10$ grid with a length of ten arbitrary units per side centred at $(5.5,5.5,0)$ is considered. Nine sensors are placed in a planar array above the grid with centre at $(5.5,5.5,2)$, see figure 11 . We use constant conductivity $\sigma$ in $\mathbb{R}^{3}$. Thus, the leadfield matrix is obtained by

$$
\begin{aligned}
& L_{i, j}=\frac{1}{4 \pi \sigma} \frac{r_{i, 1}-p_{j, 1}}{\left\|r_{i}-p_{j}\right\|^{3}} \\
& L_{i, j+N}=\frac{1}{4 \pi \sigma} \frac{r_{i, 2}-p_{j, 2}}{\left\|r_{i}-p_{j}\right\|^{3}} \\
& L_{i, j+2 N}=\frac{1}{4 \pi \sigma} \frac{r_{i, 3}-p_{j, 3}}{\left\|r_{i}-p_{j}\right\|^{3}} .
\end{aligned}
$$

Here $r_{i} \in \mathbb{R}^{3}$ is the position of the $i$ th sensor, $p_{j} \in \mathbb{R}^{3}$ is the position of the $j$ th gridpoint.

Two equally oriented dipoles with moment $(0,0,1)^{\top}$ at $x=3$, eight both at $y=5$ are placed on the $10 \times 10$ grid, see figure 11. A Gaussian dipole-strength timeseries is assigned to each dipole by

$$
q(t)=q_{0} \exp \left\{-\frac{\left(t-t_{p}\right)^{2}}{w^{2}}\right\}
$$

with peaks at time slice $t_{p}=5$ (dipole 1$)$ and $t_{p}=9$ (dipole 2) and a width of $w=2.5$ (see figure 12). 


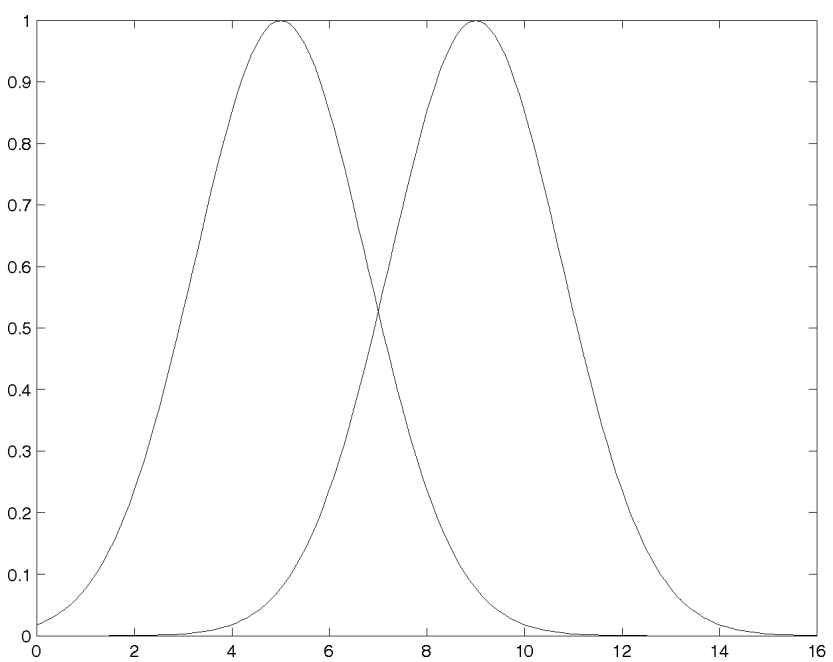

Figure 12. The activation curves of the dipoles.
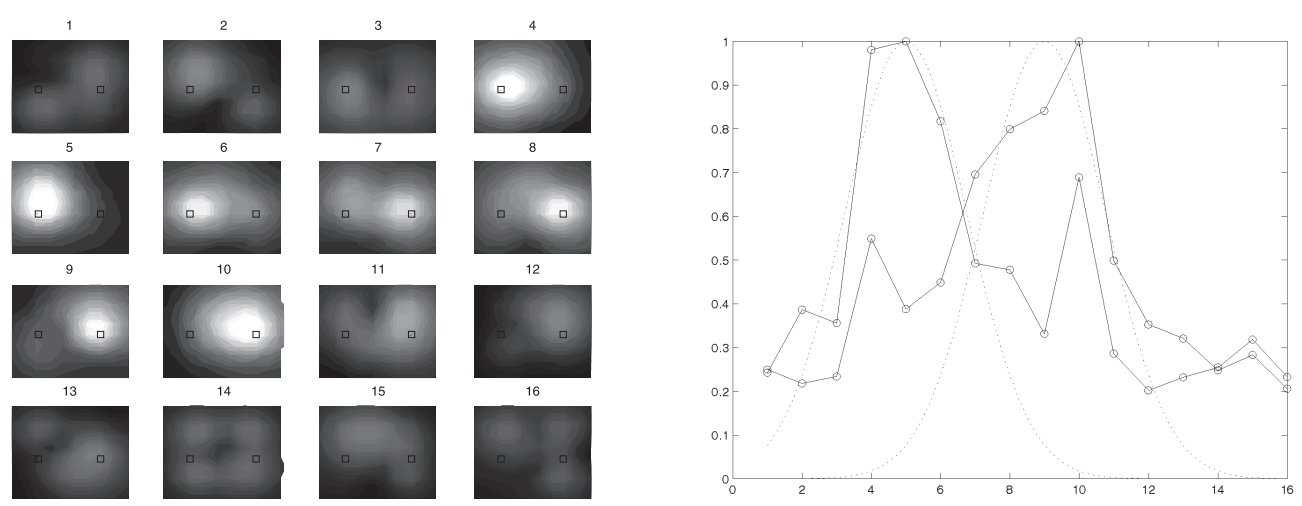

Figure 13. Temporal uncoupled reconstructions. $\lambda^{2}=2.0$.

\subsection{Numerical tests}

In the following we will compare the results of STR-C and temporal uncoupled TikhonovPhillips regularizations [27]. The latter means that $j_{t}$ is computed based on

$$
\alpha_{t}=\operatorname{minarg}_{\alpha}\left\|L \alpha-y_{t}\right\|^{2}+\lambda^{2}\|\alpha\|^{2}
$$

for each timestep $t$ and data $y_{t}, 1 \leqslant t \leqslant T$. In order to improve the signal-to-noise ratio for the uncoupled case, we used a Savitzky-Golay filter of order 3 and length 5, see [33,34].

For the following reconstructions we used synthetic data which were superposed by uniform noise in the range $\left[-0.3 \max _{t}\left|y_{t}\right|, 0.3 \max _{t}\left|y_{t}\right|\right]$. This noise range is typical for EEG measurements.

In figure 13 we see the result of the temporal uncoupled Tikhonov-Phillips procedure, in figure 14 we see the result of STR-C. In the left half of these figures the current density $\|j(p, t)\|_{\mathbb{R}^{3}}$ for each of the 16 timesteps $t$ is shown. The small boxes mark the exact positions of the dipoles. In the right half, the reconstructed activation curves are shown. 

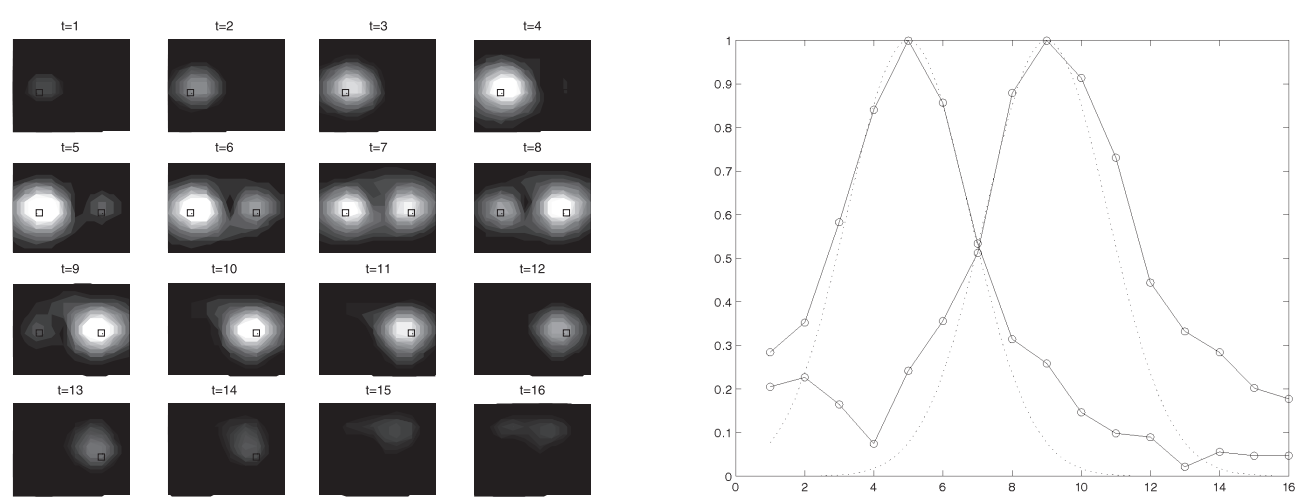

Figure 14. Reconstructions based on STR-C. $\lambda^{2}=2.0, \mu^{2}=1.5$.

\subsection{Discussion}

As one can see, our approach STR-C yields reconstructions with a smaller localization error and a more exact activation curve. Furthermore, the two dipoles are better separated. Thus, the a priori information of temporal smoothness leads to higher robustness against noise in the data.

For a systematic comparison of these approaches, based on an exactness measure for the localizations and a correlation measure for the activation curves, see the joint work [35].

If we compare our methods with the work of Brooks as proposed in [36], we start from the same model introducing temporal smoothness, but yield an immensely faster algorithm which has a significant impact on the clinical usability.

\section{Conclusion}

As shown the procedure STR is suited for the regularization of a large class of dynamic inverse problems. It was applied to dynCT which is modelled by operators between infinitedimensional spaces. Here, we could develop a procedure which is able to distinguish between pathological findings and motion artefacts.

Further dynEIT was studied, which is formulated by finite-dimensional nonlinear operators. Here, linearization lead to a practical algorithm. This algorithm provides reconstructions with a quality comparable to the reconstructions gained by so-called Kalman smoothers. In contrast to the Kalman approach which depends on three regularization parameters, our procedure only needs two such parameters which has an impact on the practical use of these procedures. Furthermore, our procedure is significantly faster and has less memory usage.

The last application to EEG source localization, namely stCDR, proved that the a priori information of temporal smoothness leads to an algorithm with higher robustness against noise than a known procedure based on temporal uncoupled Tikhonov-Phillips regularization. Furthermore, the reconstructed peaks and the reconstructed activation curves are more accurate.

Although the used a priori information of temporal smoothness is quite general, STR leads in general to reconstructions which give an insight into the temporal behaviour of the examined objects. In all applications the efficiency was outstanding. 


\section{Acknowledgments}

This work was supported by the German ministry of education and research (03-LO7SA2-2, 03-BU7AA1-4), Professor E Zeidler, director of the MPI for Mathematics in the Sciences, Leipzig and by the Leipniz-Prize of the German Research Foundation awarded to Professor A D Friederici, director of the MPI of Cognitive Neuroscience, Leipzig.

\section{References}

[1] Schmitt U and Louis A K 2002 Efficient algorithms for the regularization of dynamic inverse problems: I. Theory Inverse Problems 18 645-58

[2] Gardiner J D, Laub A J, Amato J J and Moler C B 1992 Solution of the Sylvester matrix equation $A X B^{\top}+C X D^{\top}=$ E ACM Trans. Math. Softw. $18223-38$

[3] Herman G T 1980 Image Reconstruction from Projections. The Fundamentals of Computerized Tomography (New York: Academic)

[4] Natterer F 1986 The Mathematics of Computerized Tomography (New York: Wiley)

[5] Louis A K 1989 Inverse und Schlecht Gestellte Probleme (Stuttgart: Teubner)

[6] Osterman K S et al 2000 Multifrequency electrical impedance imaging: preliminary in vivo experience in breast Physiol. Meas. 21 99-109

[7] Dijkstra A et al 1993 Review: clinical applications of electrical impedance tomography J. Med. Eng. Technol. 17 89-98

[8] Alessandrini G and Rondi L 1998 Stable determination of a crack in a planar inhomogenous conductor SIAM J. Math. Anal. 30 326-40

[9] Friedman A and Vogelius M 1989 Determining cracks by boundary measurements Indiana Univ. Math. J. 38 $527-56$

[10] Kaup P G and Vogelius M 1996 Method of imaging corrosion damage in thin plates from electrostatic data Inverse Problems 12 279-93

[11] Cheney M, Isaacson D and Newell J C 1999 Electrical impedance tomography SIAM Rev. 41 85-101

[12] Cheng G, Isaacson D, Newell J C and Gisser A 1989 Electrode models for electric current computed tomography IEEE Trans. Biomed. Eng. 36 918-24

[13] Somersalo E, Cheney M and Isaacson D 1992 Existence and uniqueness for electrode models for electric current computed tomography SIAM J. Appl. Math. 52 1023-40

[14] Kaipio J P, Kolehmainen V, Somersalo E and Vauhkonen M 2000 Statistical inversion and Monte Carlo sampling methods in statistical impedance tomography Inverse Problems 16 1487-522

[15] Vauhkonen M and Karjalainen P A 1989 A Kalman filter approach to track fast impedance changes in electrical impedance tomography IEEE Trans. Biomed. Eng. 45 486-93

[16] Vauhkonen M 1997 Electrical impedance tomography and prior information PhD Thesis University of Kuopio, Kuopio, Finland

[17] Kaipio J P, Karjalainen P A, Somersalo E and Vauhkonen M 1999 State estimation in time-varying electrical impedance tomography Ann. NY Acad. Sci. 430-9

[18] Grewal M S and Andrews A P 1993 Kalman Filtering (Englewood Cliffs, NJ: Prentice-Hall)

[19] Vauhkonen P J, Vauhkonen M and Kaipio J P 2001 Fixed-lag smoothing and state estimation in dynamic electrical impedance tomography Int. J. Numer. Methods Eng. 50 2195-209

[20] Kim K Y, Kim B S, Kim M C, Lee Y J and Vauhkonen M 2001 Image reconstruction in time-varying electrical impedance tomography based on the extended Kalman filter Meas. Sci. Technol. 12 1032-9

[21] Waberski T D, Buchner H, Herrendorf G, Gobbele R and Paulus W 2000 Properties of inverse methods in temporal lobe epilepsie Epilepsia 14 1574-83

[22] Buchner H, Waberski T D and Noth J 1996 Generators of early cortical somatosensory evoked potentials in men Recent Advances in Clinical Neurophysiology ed Kimura and Shibasaki (Amsterdam: Elsevier) pp 630-6

[23] Schmitt U, Louis A K, Darvas F, Buchner H and Fuchs M 2001 Numerical aspects of spatio-temporal current density reconstruction from EEG-/MEG-data IEEE Trans. Med. Imaging 20 314-24

[24] Andrä and Nowak 1989 Magnetism in Medicine-A Handbook (New York: Wiley)

[25] Gulrajani R M, Roberge F A and Savard P 1989 The inverse problem of electrocardiography Comprehensive Electrocardiology ed Macfarlane and Veitch Lawrie (Oxford: Pergamon) pp 237-88

[26] Srebro R 1994 Continuous current source inversion of evoked potential fields in a spherical model head IEEE Trans. Biomed. Eng. 42 997-1003 
[27] Louis A K 1992 Parametric reconstruction in biomagnetic imaging Inverse Problems in Scattering and Imaging ed M Bertero and E R Pike (Bristol: Adam Hilger) pp 156-63

[28] Fuchs M, Drenckhahn D, Wischmann H A and Wagner M 1998 An improved boundary element method for realistic volume-conductor modelling IEEE Trans. Biomed. Eng. 45 980-97

[29] Buchner H, Knoll G, Fuchs M, Rienaecker A, Beckmann R, Wagner M, Silny J and Pesch J 1997 Inverse localization of electric dipole current sources in finite element models of the human head Electroencephalogr. Clin. Neurophysiol. 102 267-78

[30] Anwander A, Kuhn M, Reitzinger S and Wolters C 2001 A parallel algebraic multigrid solver for finite element method based source localization in the human brain webpage http://www.mis.mpg.de/jump/publications.htm

[31] Wolters C, Hartmann U, Koch M, Kruggel F, Burkhardt S, Basermann A, Tuch D S and Haueisen J 1999 New methods for improved and accelerated FE volume conductor modeling in EEG/MEG-source reconstruction 4th Symp. on Computer Methods in Biomech. and Biomed. Eng. (Lisboa, Oct.-Nov. 1999) (London: Gordon and Breach) pp 489-94

[32] Wolters C, Beckmann R, Rienäcker A and Buchner H 1999 Comparing regularized and non-regularized nonlinear dipole fit methods: a study in a simulated sulcus structure Brain Topography 12 3-18

[33] Savitzky A and Golay M J E 1964 Anal. Chem. 36 1627-39

[34] Hamming R W 1983 Digital Filters 2nd edn (Englewood Cliffs, NJ: Prentice-Hall)

[35] Darvas F, Schmitt U, Louis A K, Fuchs M, Knoll G and Buchner H 2001 Spatio-temporal current density reconstructions (stCDR) from EEG/MEG-Data Brain Topography 13 192-208

[36] Brooks D H, Ahmad G F, MacLeod R S and Maratos G M 1999 Inverse electrocardiography by simultaneous imposition of multiple constraints IEEE Trans. Biomed. Eng. 46 3-17 